\title{
Feministische Klassenpolitiken in Kämpfen um soziale Reproduktion
}

Zu den Auseinandersetzungen an der Berliner Charité für mehr Personal im Krankenhaus

Die Krise der sozialen Reproduktion ist allgegenwärtig.[1] Nicht nur in feministisch-geschlechterkritischen Debatten, auch in medial artikulierten „Pflegeskandalen` oder Berichten über steigende ,Burn-Out'-Zahlen spielen Entwicklungen der sozialen Reproduktion eine zunehmend wichtige Rolle.[2] Hierbei werden in den vergangenen Jahren immer wieder Lücken der Versorgung, die Zerstörung von Fürsorglichkeit[3] und/oder subjektive Erschöpfung thematisiert. Diese werden einerseits auf Veränderungen in der geschlechtsspezifischen Arbeitsteilung und Reproduktionslücken in den Privathaushalten, andererseits auf eine profitförmige Reorganisation von ,Sorgearbeit' zurückgeführt. Die Veränderungen der sozialen Reproduktion, die sich im Zuge der Durchsetzung des Neoliberalismus vollziehen, werden demnach als krisenhafte Prozesse beschrieben, in denen tradierte Reproduktionsmodelle nicht mehr gelingen und ,Sorgelücken“ sowohl im Privaten als auch im Bereich bezahlter sozialer Dienstleistungen zunehmen. Im Prozess dieser Transformation wachsen folglich Widersprüche der sozialen Reproduktion an; zugleich werden diese verstärkt zum Ausgangspunkt gesellschaftlicher Auseinandersetzungen. Insbesondere in Bereichen bezahlter Tätigkeiten der sozialen Reproduktion lassen sich zunehmend mehr Konflikte um die Ausgestaltung von (Arbeits-)Bedingungen ebenso wie um die Folgen profitförmiger Reorganisation konstatieren (vgl. Artus et al. 2017).

Solche Konflikte sind zum Beispiel in der Krankenpflege und den Kämpfen am Berliner Universitätsklinikum Charité zu beobachten. Schon seit mehreren Jahren kämpfen die Beschäftigten hier für mehr Personal im Krankenhaus. Sie reagieren damit auf die zunehmende Arbeitsverdichtung, den damit verbundenen, enorm gestiegenen Zeitdruck und einen massiven Personalabbau in der Krankenhausversorgung der vergangenen Jahre. Darüber hinaus versuchen sie auch ihrer eigenen Erschöpfung ein Ende zu setzen (vgl. Wolf 2013). Um eine am medizinischen und pflegerischen Bedarf orientierte Personalbemessung durchzusetzen und den verschärften Bedingungen im Krankenhaus sowie der budgetorientierten (Personal-) Planung etwas entgegenzusetzen, sind sie in den vergangenen Jahren immer wieder in Auseinandersetzungen getreten. Im Sommer 2015 ist es schließlich 
nach zehn Tagen Streik gelungen, erstmals eine tarifliche Personalbemessung zu erkämpfen. Seitdem sieht der Tarifvertrag ,Gesundheitsschutz an der Charité vor, den Personalbedarf entsprechend des Pflegeaufwands und nicht mehr entlang des zur Verfügung stehenden finanziellen Budgets zu bestimmen (vgl. Kunkel 2015). Mit dem neuen Tarifvertrag haben die Beschäftigten folglich nicht nur eine Umkehrung der Personalplanung, sondern auch die Beendigung eines jahrelangen Personalabbaus erreicht. In diesem Sinne können die Auseinandersetzungen an der Charité als Meilenstein gewerkschaftlicher Kämpfe bezeichnet werden. Aber sie sind nicht nur das: In den Kämpfen ist es darüber hinaus gelungen, die für die Patient*innen menschenunwürdigen Verhältnisse sowie die mangelnde Versorgung im Krankenhaus in den Fokus der Aufmerksamkeit zu rücken. In ihren Kämpfen haben die Beschäftigten der Charité verdeutlicht, was in feministischen Debatten um eine „Krise der sozialen Reproduktion" in den vergangenen Jahren vermehrt thematisiert wird: nämlich die Lücken der Versorgung, wenn beispielsweise Patient*innen auf Krankenhausfluren, geparkt' werden müssen, weil ihre Aufsicht aufgrund von Personalmangel anderenfalls nicht gewährleistet werden könnte, oder subjektive Erschöpfung, wenn Pflegekräfte die mangelnde Versorgung von Patient*innen durch Mehrarbeit in ihrer Freizeit zu kompensieren versuchen, wobei sie zugleich selbst unter dieser Überlastung leiden.

Zu Recht können die Auseinandersetzungen der Charité-Beschäftigten sowohl aus gewerkschaftlicher als auch aus feministischer Perspektive als Speerspitze der Kämpfe um soziale Reproduktion und gegen die Zurichtungen des Neoliberalismus betrachtet werden. In einem zentralen Bereich der Sorgearbeit ist es im Zuge der Auseinandersetzung an der Charité gelungen, die Widersprüche der neoliberalen Transformation von sozialen Reproduktionsverhältnissen zu kritisieren. Damit wurde nicht nur eine tarifliche ,Entlastung durch Personalbemessung erstritten, sondern darüber hinaus wurden auch die öffentlichen Debatten mitgeprägt - bis hin zum Aufgreifen des ,Pflegenotstands' als ein zentrales Thema der Bundestagswahl 2017 und der neuen Regierungsbildung.

Und dennoch fehlt in der Deutung der Kämpfe und den Debatten um die Krise der sozialen Reproduktion eine wesentliche Dimension der Kritik. Die Veränderungen der sozialen Reproduktion und der Gesundheitsversorgung im Krankenhaus werden bisher lediglich im Hinblick auf die ökonomische Dimension betrachtet, während ihre politisch-ideologischen und subjektivierenden Dimensionen[4] aus dem Blick geraten. So ist es in den Auseinandersetzungen zwar gelungen, die neoliberale, Ökonomisierung von Krankenhäusern' und die Reorganisation und Rationalisierung von (Pflege-) Arbeit zu kritisieren. Auch die Folgen für die Versorgung von Patient*innen und die (Selbst-)Sorge der Beschäftigten konnten skandalisiert werden. Allerdings sind dabei lediglich die Veränderungen der Produktions- und Arbeitsbedingungen im Krankenhaus in den Blick geraten.

Wie im Folgenden gezeigt werden soll, ist mit dem Wandel der Rahmenbedingungen im Krankenhaus jedoch auch ein Druck auf die (gesellschaftlichen) Praxen und Vorstellungen von, Fürsorglichkeit und Pflege und darin auch auf die (Arbeits-)Gewohnheiten, Routinen und pflegerischen Selbstverständnisse der Beschäftigten entstanden. Dieser ,Anpassungsdruck resultiert einerseits aus den veränderten Bedingungen der Pflege im Krankenhaus, und wird 
andererseits zugleich auch durch veränderte Formen der Subjektivierung von Pflegekräften begleitet - wie sich beispielsweise im Diskurs der ,professionellen Pflege' als medizinisch-technischer Herausforderung und einem damit zusammenhängenden Diskurs um die ,Aufwertung von Sorgearbeit‘ zeigt. Neben der Transformation der Produktions- und Arbeitsbedingungen wird im Neoliberalismus also auch ein Verständnis von sozialer Reproduktion und Pflege(-arbeit) zu verallgemeinern versucht, das diese auf die leichter zu rationalisierenden, medizinisch-technischen Aspekte verengt, während es die emotionalen, empathisch-fürsorglichen und die schwer quantifizierbaren Tätigkeiten tendenziell abspaltet.

Folglich können die Kämpfe der Charité-Beschäftigten erst im Spannungsfeld dieser vielfältigen Veränderungen umfassend verstanden werden; nämlich nicht allein als Reaktion auf veränderte (Arbeits-)Bedingungen im Krankenhaus und als Kämpfe gegen den Kostendruck, sondern auch als Auseinandersetzungen um das (Selbst-)Verständnis der Pflege(-arbeit) und um ein Festhalten der Beschäftigten an ,tradierten Gewohnheiten pflegerischer Praxis'. Um die verschiedenen Interessen der pflegenden Beschäftigten, der Patient*innen und pflegenden Angehörigen miteinander verbinden zu können, müssen also auch die politisch-ideologischen und subjektivierenden Veränderungen von Pflege und sozialer Reproduktion in den Kämpfen in den Vordergrund gerückt und zum Gegenstand der gewerkschaftlichen und feministischen Kritik gemacht werden. Erst dadurch wird eine tatsächliche Bündnisfähigkeit geschaffen, die sich an einem umfassenden gesellschaftlichen Umbau (der sozialen Reproduktion) orientiert.

\section{Neoliberale Reorganisation von sozialer Reproduktion und die In-Wert-Setzung von Sorgearbeit im Krankenhaus}

Mit der Durchsetzung neoliberaler Programmatiken seit den 1980er Jahren geraten fordistische Arrangements der sozialen Reproduktion in die Krise, die bis dahin mit Hilfe von Privathaushalten, Ernährerlöhnen sowie sozialstaatlichen Absicherungen weitestgehend sichergestellt wurden. Dabei wird die Organisation und Bereitstellung der sozialen Reproduktion im Neoliberalismus einerseits der eigenverantwortlichen Absicherung durch die Subjekte und Familien übertragen, während zugleich sozialstaatliche Absicherungen abgebaut werden; andererseits steigt aber auch der Anteil bezahlter, professioneller Tätigkeiten der Sorgearbeit an. Die Auslagerung von Tätigkeiten der sozialen Reproduktion an professionelle Dienstleister geht mit Prozessen der In-Wert-Setzung und einem qualitativen Wandel von Sorgearbeit einher, also sowohl mit einem Wandel der materiellen Rahmenbedingungen als auch veränderten Anforderungen an die Beschäftigten in der professionellen Sorge.

In den Krankenhäusern zeigen sich die seit Jahren voranschreitende Ökonomisierung von sozialer Reproduktion und der Wandel der Rahmenbedingungen vor allem in der Umstellung des Finanzierungsmodells. Hierbei wird die Finanzierung der Krankenhäuser von bedarfsdeckenden, tagesgleichen Pflegesätzen auf diagnosebezogene Fallpauschalen, d. h. von einer kostendeckenden auf eine Finanzierung nach Pauschalen pro Behandlungsfall umgestellt. Dies bringt das Risiko mit sich, dass die Kosten des 
einzelnen Krankenhauses (pro Fall) dabei möglicherweise nicht vollständig gedeckt werden (vgl. Gerlinger/Mosebach 2009). Die Einführung des sogenannten DRG-Systems (Diagnosis Related Groups, diagnosebezogene Fallpauschalen) führt folglich zu einem permanenten Kostendruck und einem strukturellen Wettbewerb unter den Kliniken. Dies hat weitreichende Konsequenzen für die Gesundheitsversorgung und die Beschäftigten im Krankenhaus. Infolge des neuen Finanzierungsmodells konzentrieren sich die Kliniken betriebswirtschaftlich auf Kostensenkungen, die sie einerseits durch Verschlechterungen in der Versorgung (wie der Steigerung der ,Fälle‘ bei kürzerer Verweildauer) und andererseits durch Personalkürzungen und Outsourcing in Pflege und Service zu erreichen versuchen. Zugleich wird die (Pflege-)Arbeit über neoliberale Steuerungsinstrumente so reorganisiert, dass der (finanzielle und zeitliche) Druck an die Beschäftigten weitergegeben wird und eine Verdichtung und Rationalisierung der Arbeit nach sich zieht. Schließlich lenkt das Abrechnungssystem der DRGs die Aufmerksamkeit darüber hinaus vor allem auf quantifizierbare, abrechenbare und daher insbesondere ,medizinisch-technische ' Tätigkeiten und führt somit auch zu einer inhaltlichen Veränderung von Pflege. Einerseits erhalten patient*innenferne Tätigkeiten darin ein höheres Gewicht, wie beispielsweise die zunehmende Dokumentationspflicht sowie die Codierung von DRGs, da auf ihnen die Abrechnung der Behandlungen basiert. Andererseits werden aber auch ärztliche und technische Aspekte der Arbeit in den Vordergrund gerückt, während zeit- und zuwendungsintensive, das heißt stärker pflegerische Aspekte als Hilfs- und Zuarbeiten an weniger qualifizierte Assistenz- und Servicekräfte delegiert werden.[5] Zugleich steigt der Druck, Diagnosen und Therapien zu standardisieren, das heißt sie nicht mehr den individuellen Bedürfnissen der Patient*innen anzupassen, sowie abweichende, pflegeaufwendigere und weniger gewinnbringende Behandlungsmethoden möglichst zu minimieren. Letztlich erscheint die Pflege also nur noch als Erbringerin von technischen und medizinischen, standardisierten Abläufen, während die individuelle Zuwendung des pflegenden Personals an die Patient*innen auf der Strecke bleibt.

Durch die Umstellung des Finanzierungsmodells geraten die Krankenhäuser also einerseits in einen permanenten Kosten- und Konkurrenzdruck, den sie mit Hilfe von Personalabbau, Arbeitsverdichtung und Rationalisierung zu bearbeiten versuchen. Zugleich verschieben sich andererseits jedoch auch die Bedeutung der verschiedenen Dimensionen pflegerischer Arbeit sowie die Anforderungen an die Beschäftigten, da medizinisch-technische und standardisierte Abläufe sowie Dokumentationen und Codierungen gewinnbringender DRGs an Bedeutung gewinnen. Die Reorganisation der Gesundheitsversorgung im Krankenhaus ist demnach letztlich als umfassende Neu-Formierung zu verstehen, denn darin werden sowohl bestehende Produktions- und Arbeitsbedingungen als auch das tradierte, gesellschaftliche Verständnis und Praxen der Pflege und Fürsorge umgewälzt. Die Zunahme bezahlter, professioneller Sorge sowie ihre Reorganisation im Neoliberalismus, die sich im Krankenhaus wesentlich durch die Abkehr von der bedarfsdeckenden Finanzierung zu einer Finanzierung nach Fallpauschalen vollzieht, geht demnach nicht nur mit einem Wandel der Form, sondern auch des Inhalts von sozialer Reproduktion einher. 


\section{Der Kampf der Charité-Beschäftigten für mehr Personal: Ökonomisierung und Kostendruck in der Kritik - für mehr Fürsorglichkeit?}

Mit der Veränderung der Rahmenbedingungen sowie der darin implizierten Vorstellungen von Pflege geraten auch die(Arbeits-)Gewohnheiten, alltäglichen Routinen und (pflegerischen) Selbstverständnisse der Beschäftigten unter Druck. Denn einerseits werden in der betriebswirtschaftlichen Orientierung auf Kostensenkungen die Rahmenbedingungen so verändert, dass die Möglichkeiten für eine fürsorgliche Praxis eingeschränkt werden. Es fehlt also schlichtweg die Zeit für eine umfassende Versorgung der Patient*innen. Andererseits wird durch die implizite Re-Formulierung des Pflegeverständnisses in den DRGs und ihre Orientierung an Profitabilität die pflegerische Routine zu Veränderungen gezwungen; denn die Versorgung auf höchstem technischen und medizinischen Niveau, wachsende Dokumentationstätigkeiten oder das immer schnellere ,Durchschleusen“ von Patient*innen werden darin wichtiger als die Körperpflege, das fürsorgliche Gespräch oder emotionale Anteilnahme. Während also Dokumentationen oder (teilweise an die Pflege übertragene) ärztliche Tätigkeiten auch unter Zeitdruck nicht verschoben werden können, werden individuelle Ängste oder Bedürfnisse der Patient*innen - und damit eigentlich ein Kernanliegen pflegerischer Arbeit zur Manövriermasse, die erledigt werden kann, wenn alles andere getan ist. Mit der Einführung der DRGs wird folglich das „Ethos fürsorglicher Praxis“[6] (Senghaas-Knobloch 2008: 227), das langeZeit nicht nur das Selbstverständnis der Beschäftigten prägte, sondern auch den Anforderungen an die pflegerische Praxis entsprach, in Frage gestellt. Die veränderten Bedingungen im Krankenhaus setzen die tradierte, pflegerische Praxis der Beschäftigten folglich in doppelter Weise unter Druck: Während die Bedingungen fürsorglicher Versorgung massiv eingeschränkt werden, wird auch das Verständnis ,guter Pflege' reformuliert, indem den medizinisch-technischen, standardisierten und abrechenbaren Tätigkeiten Vorrang eingeräumt wird.

Vor dem Hintergrund von Zeitdruck und einem Fokus auf gewinnträchtige Tätigkeiten kann dieser Verlust empathisch-emotionaler Zuwendungen nur mit Hilfe von individueller (meist unbezahlter) Mehrarbeit der Beschäftigten, das heißt nur auf Kosten der eigenen Gesundheit und zum Preis von Erschöpfung kompensiert werden. Das Festhalten an einem ,Ethos fürsorglicher Praxis' entgegen den gewandelten Anforderungen an die Beschäftigten zieht also Überstunden, Überlastung und Erschöpfung nach sich.[7] Aber auch ohne die Versuche individueller Kompensation führen Personalmangel und Zeitdruck bei den Beschäftigten zu Überforderung und Erschöpfung. Folglich geraten die Pflegekräfte in einen Konflikt zwischen Personalabbau, Arbeitsverdichtung, Zeitdruck und veränderten Anforderungen an die pflegerische Arbeit auf der einen und einem tradierten, auf geduldige, empathische und zugewandte Pflege setzenden Selbstverständnis auf der anderen Seite. Letzteres prägt zumindest teilweise noch den Anspruch an die eigene Arbeit. Die Pflegekräfte finden sich dabei immer in der Überlastungsfalle wieder, ganz egal, für welche Seite sie sich entscheiden.

Dass die Beschäftigten der Charité in dieser Situation eine verbindliche Personalbemessung einfordern, kann als Reaktion auf diese Gemengelage 
verstanden werden. Aufgrund von Erschöpfung und unbezahlten Überstunden, der Verdichtung und Rationalisierung der Arbeit sowie der Missachtung eines weiblich* konnotierten ,Pflegeethos' klagen sie (Arbeits-) Bedingungen ein, die es erlauben, den Bedürfnissen der Patient*innen und dem eigenen Anspruch der Pflegekräfte an ihre Arbeit gerecht zu werden. In diesem Sinne prägt auch der Slogan: „Mehr von uns ist besser für alle“ die Kämpfe der Beschäftigten. Er verweist auf diese doppelte Perspektive, indem er sowohl die Überforderung der Pflegekräfte ernst nimmt, als auch die Versorgungssituation der Patient*innen aufgreift, die unter Personalmangel und den damit einhergehenden Folgen zu leiden haben. ,Erschöpfung' und ,Qualitätsverlust der Versorgung' sind demnach zwei Motive, die in den Auseinandersetzungen immer wieder artikuliert und in Zusammenhang gebracht werden. Dabei wird aufgezeigt, dass aufgrund des Personalmangels keine Zeit mehr für Gespräche und emotionale Zuwendung bleibt, sodass die Pflege lediglich in einem medizinisch-technischen Sinne Leben erhält. Personalbemessung wird folglich zu einem Instrument gegen die Verschärfung der Arbeitsbedingungen auf der einen und für eine ,gute Versorgung' auf der anderen Seite.

Die Forderung nach einer verbindlichen Personalbemessung stellt also definitiv eine Antwort auf die veränderten Arbeitsbedingungen und eine Verbesserung der Versorgung dar - insofern ,mehr Pflege immer auch eine Entlastung für die Pflegekräfte und eine Voraussetzung für eine gute Versorgungssituation ist. Jedoch verteidigt sie darin nicht notwendiger Weise auch ein ,Ethos fürsorglicher Praxis'. Denn welche Pflege mit der Forderung nach mehr Personal ermöglicht werden soll, ist mit der doppelten Strategie der Zurückweisung von Erschöpfung sowie der Schaffung ,guter Pflegebedingungen noch nicht umschrieben.

\section{Aufwertung von Sorgearbeit und hegemoniale Einbindung: Verschiebungen im Ethos fürsorglicher Praxis}

Da sich mit der Einführung der DRGs die Praxis pflegerischer Arbeit verschiebt, gerät darin auch ein Selbstverständnis in die Krise, welches Fürsorge, Zuwendung und emotionale Bezugnahme als Kernbestandteil der pflegerischen Arbeit begreift. Die veränderten Bedingungen im Krankenhaus und die Reformulierung des Pflegeverständnisses führen bei den Beschäftigten nicht nur zu Zeitdruck und einer Verschiebung pflegerischer Tätigkeiten. Sie bewirken auch einen ,Anpassungsdruck' auf das Selbstverständnis und die Ansprüche der Pflegekräfte an ihre eigene Arbeit. Dieser Anpassungsdruck entsteht nicht nur durch die veränderten Rahmenbedingungen, sondern kommt beispielsweise auch im gesellschaftlichen Diskurs um eine ,Aufwertung und Professionalisierung der Sorgearbeit' zum Tragen. Auch hierin wird gegenwärtig das Verständnis von Pflege reformuliert.

Im Diskurs um ,Aufwertung“ werden demnach Hoffnungen auf eine Zunahme gesellschaftlicher Wertschätzung geschürt. Dies geht allerdings Hand in Hand mit einem Weg der Pflege in die Sozialwirtschaft, bei der sie ihre historisch bedingte Sondersituation verlässt. Während mit der verstärkten In-Wert-Setzung auch eine größere ökonomische Bedeutung der Pflege einhergeht, ebnet der ,Formwandel ${ }^{`}$ der Pflege dabei zugleich den 
Weg für ihre Anerkennung als ,echte Profession'. In-Wert-Setzung und ,Professionalisierung bilden die zwei Seiten der ,Aufwertung' des Berufsfeldes der Pflege. Einerseits ist die Sozialwirtschaft in den vergangenen anderthalb Jahrzehnten auf einem Wachstumspfad, der sich an einem massiven Anstieg der Beschäftigtenzahlen sowie der Bruttowertschöpfung bemerkbar macht und damit die Grundlage für ihre zunehmende ökonomische Bedeutung bildet.[8] Andererseits bietet die Professionalisierung der Pflege die Chance, diese gesellschaftlich notwendige Arbeit aufzuwerten, indem sie endgültig aus den Haushalten herausgeholt und sichtbar gemacht wird und mit wachsenden Ansprüchen an die Professionalität und Qualifizierung der Beschäftigten sowie der Zurückweisung einer Vorstellung einhergeht, nach der praktisch jede*r Sorgearbeit leisten kann. Damit kommt der ,Sorgearbeit jedoch nicht nur eine wachsende gesellschaftliche Bedeutung zu. Vielmehr wird durch Prozesse der ,Professionalisierung auch eine spezifische Form der Aufwertung bestärkt. So wird ein Deutungsangebot formuliert, welches die bisherige Abwertung der Sorgearbeit damit erklärt, dass sie mit der Last der Traditionen zu kämpfen habe, nämlich damit, dass Arbeit im Sozialsektor teils immer noch eher als selbstlose Barmherzigkeit denn als professionelle Berufstätigkeit aufgefasst werde (vgl. Blank/Schulz/Voss 2017: 172). Die Zurückweisung der Sorgearbeit als wenig qualifizierte und wertvolle Arbeit und ihre Professionalisierung als Befreiung von ebendiesen ,Altlasten' stellt die Voraussetzung für eine echte, gesellschaftliche Aufwertung dar.[9] Zugleich wird darin jedoch auch ein weiblich* konnotiertes, fürsorglich-selbstloses und zugewandt-emotionales Arbeitsethos in Frage gestellt. Denn die ,Befreiung aus einer lange währenden Abwertung vollzieht sich sogleich auf Kosten von bis dahin der Pflege zugesprochenen Charakteristika und Tätigkeiten.[10] Parallel zur Bedeutungsverschiebung pflegerischer Tätigkeiten durch die DRGs wird im Aufwertungsdiskurs, wie oben dargelegt, ein medizinischtechnisches, ,professionelles ${ }^{\varsigma}$ und neoliberal rationalisierbares Verständnis von Pflege stark gemacht.[11] Was daran aufhorchen lässt, ist jedoch nicht, dass die Ausweitung des pflegerischen Tätigkeitsbereichs, das Erlernen neuer Fähigkeiten oder die fachliche Erweiterung der Pflege an sich positiv aufgegriffen werden, sondern dass dies in Abgrenzung zu einer scheinbar überkommenen Vorstellung ,fürsorglicher Pflege' geschieht.

Dass der (Anpassungs-)Druck, der auf das pflegerische (Selbst-)Verständnis wirkt, tatsächlich zu einer gesellschaftlichen Veränderung des Pflegeverständnisses führen könnte, zeigt sich nicht zuletzt daran, dass selbst dort, wo gegenwärtig noch Kämpfe gegen die neoliberalen Zurichtungen organisiert werden, das Verständnis ,guter Pflege umkämpft ist. Denn obwohl in den Auseinandersetzungen an der Charité immer wieder der Anspruch formuliert wurde, ,gute Pflege' leisten und dem eigenen Anspruch genügen zu wollen, wurde zugleich (wenn auch eher vereinzelt) ebenfalls betont, dass das Bild einer sich für andere jederzeit aufopfernden Krankenschwester aufzugeben sei, da in diesem Verständnis der Pflege eine wesentliche Ursache der Überlastung liege. Stattdessen wurde demgegenüber ein medizinischtechnisches und damit klarer abgrenzbares Verständnis pflegerischer Arbeit in Stellung gebracht und darauf verwiesen, dass Krankenpflege eben nicht „allein als das Betüdeln von Patienten“ missverstanden werden sollte, da die alltägliche Arbeit vielmehr einer medizinisch-technischen Fertigkeit 
bedürfe.[12] Folglich wurde auch in den Auseinandersetzungen an der Charité das Bild der aufopfernden, fürsorglichen und liebesdienstleistenden Krankenschwester zurückgewiesen. Dies geschah nicht nur aufgrund der Verausgabung in unbezahlten Überstunden und der Überlastung, sondern auch, da die Veränderung der Pflege als begrüßenswerte inhaltliche Verschiebung erscheint. Dass sich der Schwerpunkt pflegerischer Praxis von einer an den individuellen Bedürfnissen der Patient*innen orientierten, empathisch-emotionalen Versorgung weg verschiebt, wird folglich nicht zurückgewiesen, sondern - zumindest teilweise - positiv mitvollzogen. Es scheint so, als hätte sich die Reformulierung von Pflege zumindest vereinzelt bereits in ein neues pflegerisches Selbstverständnis eingeschrieben, nämlich eines, das auf professionell kategorisierbare Aspekte und einen medizinischtechnischen Pflegeprozess rekurriert.[13]

Die Reorganisation der Gesundheitsversorgung im Krankenhaus und die Bedeutungsverschiebung von Pflege werden folglich nicht allein durch eine negative Begrenzung des Handlungsspielraums von Pflegekräften, sondern ebenso durch ihre (scheinbare) Befreiung aus alten Traditionen verfolgt. Dies betrifft eine Erweiterung des pflegerischen Tätigkeitsbereichs und das Versprechen der Überwindung einer jahrzehntelangen Abwertung von Sorge - letzteres aber nicht durch Aufwertung, sondern durch eine Auslagerung ,fürsorglicher Tätigkeiten aus der eigentlichen Pflege.

\section{Politischer Ausblick: Die Notwendigkeit einer aktiven Herstellung von, guter Pflege ${ }^{6}$ als gemeinsamer Fluchtpunkt}

Der Blick auf die neoliberale Reorganisation der sozialen Reproduktionsverhältnisse am Beispiel der Gesundheitsversorgung im Krankenhaus zeigt, wie umfassend die Veränderungen sind, die gegenwärtig im Bereich der Sorgearbeit stattfinden. Nicht nur die Formen der sozialen Reproduktion - wie die Zunahme bezahlter Sorgearbeit, ihre Rationalisierung, Zeitdruck und Arbeitsverdichtung - wandeln sich; es verändern sich auch ihre Inhalte. Während die Veränderung der Rahmenbedingungen gegenwärtig jedoch auf Widerspruch trifft, scheinen sich die politischen und ideologischen Veränderungen der sozialen Reproduktion fast unbemerkt verallgemeinern zu können. Sie stoßen teilweise sogar auf Zuspruch. Insofern die Forderung nach mehr Personal die für die Beschäftigten zentral entstehenden Widersprüche neoliberaler Transformation adressiert, stellt sie dabei zwar nicht nur einen legitimen, sondern auch einen taktisch richtigen Schritt im Kampf um die Zurückdrängung von Arbeitsverdichtung, Zeitdruck und Reorganisation der Arbeit im Krankenhaus dar; was derzeit fehlt ist jedoch die Formierung eines Interesses an ,guter ' im Sinne von empathisch-emotionaler Fürsorge. Gegenwärtig werden die Kämpfe allerdings wesentlich von Seiten der Pflegekräfte vorangetrieben, während ein (gesellschaftliches) Interesse an guter und fürsorglicher Pflege bislang nur vereinzelt von Patient*innen, pflegenden Angehörigen oder potenziellen Patient*innen artikuliert wird.

Für feministische Klassenpolitiken stellt sich daher die Herausforderung, auch die politisch-ideologischen Veränderungen in den Fokus der Kritik zu rücken - und das nicht nur, weil die Abspaltung, das Unsichtbarmachen und die Delegation fürsorglicher Aspekte in unentlohnte Bereiche schon 
immer Themen feministischer Auseinandersetzungen waren, sondern auch, weil sich über die Abspaltung nicht rationalisierbarer, fürsorglicher Aspekte ein neoliberales Reproduktionsmodell zu stabilisieren droht. So ist die Forderung nach ,mehr Personal' angesichts der Verschärfung von Arbeits- und Pflegebedingungen im Zuge neoliberaler Transformationen zwar durchaus ein Angriff gegen diese. Dennoch ist denkbar, dass das Interesse an Personalbemessung aufgegriffen wird, ohne dass dies zu einer Infragestellung der veränderten Produktionsweise im Krankenhaus führen muss. Denn der dadurch zusätzlich entstehende Kostendruck könnte beispielsweise auch durch eine verstärkte Spaltung der Belegschaften, eine selektive Aufstockung von Personal, die Delegation von angeblich weniger qualifizierter Arbeit usw. bearbeitet werden. Und dies könnte auf der Grundlage eines veränderten Pflegeverständnisses legitimiert werden, nach welchem examinierte Pflegekräfte durch Hilfskräfte von den, dreckigen' und scheinbar unqualifizierten, weiblich* konnotierten Tätigkeiten befreit werden können und sollen. Tendenzen in diese Richtung lassen sich bereits beobachten.

Die Formierung eines Interesses an einer fürsorglich-empathischen Pflege ist daher zentral für eine gemeinsame Auseinandersetzung von Pflegekräften, Patient*innen und potenziellen Patient*innen. Diese Verknüpfung verschiedener Interessen muss aber aktiv hergestellt werden, denn sie ergibt sich nicht widerspruchsfrei aus dem Kampf für mehr Personal. Das jedoch ist nicht die Aufgabe der streikenden Pflegekräfte, sondern einer feministischklassenpolitischen Linken, der es darum gehen sollte, die Kritik an der neoliberalen Reorganisation sozialer Reproduktion zum Ausgangspunkt eines umfassenden Umbaus der Gesellschaft zu machen.

\section{Endnoten}

[1] Dieser Text ist in Zusammenarbeit mit Julia Garscha entstanden, mit der ich nicht nur die wertvollen Erfahrungen in der Bündnisarbeit „Berlinerinnen und Berliner für mehr Personal im Krankenhaus“ sowie in den Streiks an der Charité teile, sondern auch viele Diskussionen rund um die hier aufgeworfenen Fragen. Danke für die vielen gemeinsamen Abende beim Stammtisch und besonders für die Arbeit an diesem Artikel!

[2] Mit sozialer Reproduktion bezeichne ich diejenigen Prozesse, welche die physische, psychische und generative ebenso wie die subjektivierende Reproduktion der Arbeitskraft gewährleisten. Sie werden mit Hilfe von hierarchischen Geschlechterverhältnissen reguliert und sind historisch je spezifisch organisiert.

[3] Mit der These tief greifender Erosionskrisen fordistischer Arrangements seit Mitte der 1970er Jahre ist die Annahme verbunden, dass das gesamte fordistische Institutionengefüge von Kleinfamilie, Ernährer- und Hausfrauenmodell sowie Wohlfahrtsstaat erodiert ist und zu einem veränderten, dem Kapitalismus eigenen Verhältnis von Verwertung und Existenzsicherung beigetragen hat, im Zuge dessen die öffentliche Daseinsfürsorge unter dem Vorzeichen der Markteffizienz in ihrer Funktionsfähigkeit für die individuelle und gesellschaftliche Reproduktion gefährdet wurde. Diese Entwicklungen der sozialen Reproduktion bezeichnet Brigitte Aulenbacher als Zerstörung von Fürsorglichkeit, die aus ihrer Perspektive zu einer Reproduktionskrise der Gesellschaft führt (Aulenbacher 2009: 64ff.).

[4] Mit den politisch-ideologischen und subjektivierenden Dimensionen sind zivilgesellschaftliche und staatliche Veränderungen gemeint, die auf die Formierung von Subjekten und somit ihre Denk-, Fühl- und Handlungsweise einwirken.

[5] So werden beispielsweise zunehmend ärztliche Tätigkeiten auch Pflegekräften abverlangt, während zugleich bestimmte Aspekte pflegerischer Arbeit, die häufig als weniger voraussetzungsvoll angesehen werden, wie beispielsweise das Ausgeben von Mahlzeiten 
oder Hilfestellungen bei der Körperpflege, ausgelagert und an schlechter bezahltes Personal oder ,ehrenamtliche‘, pflegende Angehörige delegiert werden (vgl. Roth 2011).

[6] Mit dem Begriff „Ethos fürsorglicher Praxis“ beschreibt Eva Senghaas-Knobloch besondere Anforderungen an und Haltungen von Pflegekräften, die sich daraus ergeben, dass pflegebedürftige Menschen in besonderer Weise abhängig von ihnen sind. Die sensible Beachtung dieser Abhängigkeitskonstellation und der Anspruch der Aufrechterhaltung „der Würde des bedürftigen Individuums“ (Senghaas-Knobloch 2008: 227) vor dem Hintergrund dieser asymmetrischen Beziehung ist dabei wesentlicher Bestandteil des Ethos. Unter fürsorglicher Praxis versteht sie die vielfältigen Tätigkeiten, auf die Menschen in verschiedenen Phasen ihres Lebens existenziell angewiesen sind.

[7] Auch wenn das Festhalten am ,Ethos fürsorglicher Praxis‘ zu Erschöpfungen führen kann, ist nicht gesagt, dass die Anpassung an veränderte Erfordernisse davor bewahrt. Denn auch das Einfügen in veränderte Anforderungen kann Unzufriedenheiten und Überforderungen nach sich ziehen, wenn das zur Folge hat, dass der Anspruch an die eigene Arbeit nicht realisiert werden kann und daher zu einem ,Leiden an den Verhältnissen' führt.

[8] So sind zwischen 2000 und 2014 mehr als 1,3 Millionen neue Arbeitsplätze entstanden und die Bruttowertschöpfung ist zwischen 2000 und 2012 um rund 30 Prozent auf insgesamt 165 Milliarden Euro gestiegen. Darüber hinaus legen alle Prognosen nahe, dass ,Sorgearbeit‘ in ihrer quantitativen Bedeutung künftig eine weitere Aufwertung erfahren wird (vgl. Evans/Kerber-Clasen 2017: 181).

[9] Interessant ist dabei, dass parallel zu den Veränderungen in der Pflege und dem pflegenden Selbstverständnis auch die im Zuge des Neoliberalismus veränderten Formen weiblicher Subjektivierung in eine ähnliche Richtung weisen: Vor dem Hintergrund einer Verschiebung des Idealbilds hegemonialer Weiblichkeit in Richtung der erfolgreichen, hochqualifizierten und karrierebewussten Managerin von Familie und Beruf scheint zumindest ein Teil der unbegrenzten, häuslichen Verausgabung für das Wohl und die Fürsorge der ,Liebsten ' abgeschrieben zu sein; die teilweise Abkehr von ,Fürsorglichkeit‘ ist also en vogue. Neoliberale Weiblichkeit setzt daher auf eine partielle Infragestellung weiblicher Zuständigkeit für Sorgeverpflichtungen zugunsten einer erst dadurch ermöglichten Ausübung des Berufs.

[10] Bemerkenswert ist daran besonders, dass der Verweis auf die ,Unsichtbarmachung“ weiblicher Qualifikationen lange Zeit allein in feministischen Auseinandersetzungen Thema war, diese im Aufwertungsdiskurs jedoch aufgegriffen und spezifisch überformt werden.

[11] So dominieren quantifizierbare, scheinbar objektive Daten und maschinenlogische Wissensformen den derzeitigen Professionalisierungsdiskurs, während andere, nicht sichtbare und schlecht messbare Anteile pflegerischer Arbeit als ,nichtprofessionelle Wissenstypen' abgewertet werden. Zugleich schließt die evidenzbasierte Praxis dabei nahtlos an die durch das DRG-System beförderten Konzepte wie die Diagnose- und Klassifikationssysteme, klinische Behandlungspfade und Qualitätsmanagementprogramme an (vgl. Friesacher 2008: 4).

[12] Das Zitat sowie die Einschätzung der Ambivalenzen in Bezug auf das ,Pflegeethos` der Pflegekräfte speisen sich aus meinen politischen Aktivitäten im Bündnis „Berlinerinnen und Berliner für mehr Personal im Krankenhaus“, welches die Auseinandersetzungen an der Charité langjährig begleitet und unterstützt hat. Diese Beobachtungen und deren Interpretationen ersetzen keine (noch fehlende) detaillierte empirische Auswertung, sondern dienen vor allem der Annäherung und Illustration meiner Thesen.

[13] Dies bleibt letztlich eine empirische Frage, die im Rahmen dieses Artikels nicht weiter verfolgt werden kann. Hier geht es mir zunächst erst einmal darum, zu reflektieren, welche Gefahren eine Vernachlässigung der ideologischen Veränderungen von Pflege birgt unabhängig davon, ob sie sich bereits in ein neues Selbstverständnis eingeschrieben haben oder in den Kämpfen (bisher noch) abgewehrt werden können. 


\section{Autor_innen}

Julia Dück ist Politikwissenschaftlerin und Soziologin. Sie beschäftigt sich mit marxistischer und feministischer Gesellschaftstheorie, Krise, sozialer Reproduktion, Geschlechterverhältnissen und Care.

juliadueck@web.de

\section{Literatur}

Aulenbacher, Brigitte (2009): Arbeit, Geschlecht und soziale Ungleichheiten. Perspektiven auf die Krise der Reproduktion und den Wandel von Herrschaft in der postfordistischen Arbeitsgesellschaft. In: Arbeits- und Industriesoziologische Studien 2/2, 61-78.

Artus, Ingrid / Birke, Peter / Kerber-Clasen, Stefan / Menz, Wolfgang (2017): Die aktuellen Kämpfe um Sorge-Arbeit. Zur Einleitung. In: Dies. (Hg.), Sorge-Kämpfe. Auseinandersetzungen um Arbeit in sozialen Dienstleistungen. Hamburg: VSA Verlag, 7-33.

Blank, Florian / Schulz, Susanne Eva / Voss, Dorothea (2017): Erwerbstätigkeit im Sozialund Gesundheitssektor: ausgebaut, aufgewertet, ausgebremst? In: WSI Mitteilungen 3, Editorial, 172.

Evans, Michaela / Kerber-Clasen, Stefan (2017): Arbeitsbeziehungen in der Care-Arbeit: Blockierte Aufwertung? In: WSI Mitteilungen 3, 180-188.

Friesacher, Heiner (2008): Theorie und Praxis pflegerischen Handelns. Begründung und Entwurf einer kritischen Theorie der Pflegewissenschaft. Osnabrück: V\&R.

Gerlinger, Thomas / Mosebach, Kai (2009): Die Ökonomisierung des deutschen Gesundheitswesens. In: Nils Böhlke / Thomas Gerlinger / Kai Mosebach / Rolf Schmucker / Thorsten Schulten (Hg.), Privatisierung von Krankenhäusern: Erfahrungen und Perspektiven aus Sicht der Beschäftigten. Hamburg: VSA Verlag, 10-40.

Kunkel, Kalle (2016): Kampf gegen die Burnout-Gesellschaft in Zeiten der Digitalisierung. Der Tarifkonflikt an der Charité für Gesundheitsschutz und Mindestbesetzung. In: Lothar Schröder / Hans-Jürgen Urban (Hg.), Jahrbuch Gute Arbeit 2016. Digitale Arbeitswelt Trends und Anforderungen. Frankfurt: Bund Verlag, 253-266.

Roth, Ines (2011): Die Arbeitsbedingungen in Krankenhäusern aus Sicht der Beschäftigten. Ein Branchenbericht auf Basis des DGB-Index Gute Arbeit. Herausgegeben von Ver.di - Vereinte Dienstleistungsgewerkschaft, Berlin. https://innovation-gute-arbeit.verdi. de/++file++53edcf1d6f6844064a0o0492/download/Arbeitsbedingungen\%20in\%20 Krankenh\%C3\%A4usern.pdf (letzter Zugriff am 1.4.2018)

Senghaas-Knobloch, Eva (2008): Care-Arbeit und das Ethos fürsorglicher Praxis unter neuen Marktbedingungen am Beispiel der Pflegepraxis. In: Berliner Journal für Soziologie 18/2, 221-243.

Wolf, Luigi (2013): „Patienten wegstreiken“ - Arbeitskämpfe an der Charité. In: Zeitschrift Luxemburg. Gesellschaftsanalyse und linke Praxis 15/1, 58-63. 
